\title{
Differential DNA Methylation is associated with Hippocampal Abnormalities in Pediatric Posttraumatic Stress Disorder
}

Judith Ensink

Amsterdam University Medical Center

Taylor Keding

University of Wisconsin School of Medicine \& Public Health

Peter Henneman

Amsterdam University Medical Center

Andrea Venema

Amsterdam University Medical Center

Ligia Papale

University of Wisconsin School of Medicine

\section{Reid Alisch}

University of Wisconsin School of Medicine https://orcid.org/0000-0003-0637-4137

\section{Yousha Westerman}

Amsterdam University Medical Center

Guido van Wingen

Academic Medical Center https://orcid.org/0000-0003-3076-5891

Jasper Zantvoord

Amsterdam University Medical Center https://orcid.org/0000-0002-6475-902X

Christel Middeldorp

University of Queensland https://orcid.org/0000-0002-6218-0428

\section{Marcel Mannens}

Amsterdam University Medical Center

Ryan Herringa ( $\square$ herringa@wisc.edu )

University of Wisconsin-Madison https://orcid.org/0000-0002-1936-7959

\section{Ramón Lindauer}

Amsterdam University Medical Center

\section{Article}

Keywords: child trauma, maltreatment, pediatric PTSD, epigenetics, children, adolescents, methylation

Posted Date: October 23rd, 2020 
DOI: https://doi.org/10.21203/rs.3.rs-87419/v1

License: () (i) This work is licensed under a Creative Commons Attribution 4.0 International License. Read Full License

Version of Record: A version of this preprint was published at Biological Psychiatry: Cognitive Neuroscience and Neuroimaging on May 1st, 2021. See the published version at https://doi.org/10.1016/j.bpsc.2021.04.016. 


\section{Abstract}

Recent findings in neuroimaging and epigenetics offer important insights into brain structures and biological pathways of altered gene expression associated with posttraumatic stress disorder (PTSD). However, it is unknown to what extent epigenetic mechanisms are associated with PTSD and its neurobiology in youth. Therefore, we combine genome-wide epigenetic and structural neuroimaging measures in a Dutch cohort of youth with PTSD (ages 8-18 years). We aimed to replicate findings in a similar independent American cohort. We found significant methylome-wide associations for pediatric PTSD (FDR $p<0.05$ ) compared to non-PTSD control groups (traumatized and non-traumatized youth).

Methylation differences on 9 genes were replicated, including genes related to glucocorticoid functioning. In both cohorts, methylation on OLFM3 gene was further associated with anterior hippocampal volume. These findings point to molecular pathways involved in inflammation, stress response, and neuroplasticity as potential contributors to neural abnormalities and provide potentially unique biomarkers and treatment targets for pediatric PTSD.

\section{Main Text}

Childhood trauma is common and imposes a substantial psychological burden on youth. Approximately two-thirds of youth are exposed to psychological trauma before they reach adulthood, and up to $16 \%$ of all youth develop post-traumatic stress disorder (PTSD) by the age of 18 (Alisic et al., 2014; McLaughlin et al., 2013). Youth suffering with PTSD often show lower academic achievement than their non-affected counterparts; this decrease in achievement is coupled with increased rates of depression, suicide attempts, and substance abuse into adulthood (Copeland, Keeler, Angold, \& Costello, 2007; Panagioti, Gooding, Triantafyllou, \& Tarrier, 2015). These statistics highlight the need for additional research into the neurodevelopmental underpinnings of pediatric PTSD, with the goals of improving detection, prognosis, and treatment.

The developmental origins of health and disease hypothesis posits that early life exposures, including exposure to childhood trauma, will have prolonged effects on child health, including neurodevelopmental outcomes. Epigenetic alterations provoked by exposure to childhood trauma are likely to play a central role in the molecular mechanisms underlying the emergence of PTSD in youth. Specifically, trauma-related alterations in DNA methylation (DNAm) at cytosine-guanine junctions (CpGs) may influence the developmental programming of neural circuitry underlying stress responses, including the hippocampus, amygdala, and medial prefrontal cortex (mPFC) (Walton, Calhoun, Heijmans, Thompson, \& Cecil, 2020).

Important strides have been made in identifying epigenetic substrates of adult PTSD using peripheral DNAm markers (Smith et al., 2019). Other studies have also reported associations between DNAm and brain phenotypes in predominantly healthy cohorts (Jia et al., 2019; Lin et al., 2018; Liu et al., 2015; Ruggeri et al., 2015). However, despite these promising results, it remains unclear whether similar DNAm markers are present in a pediatric PTSD, which is of critical importance given ongoing physiological and neurodevelopment in youth. It is also unclear whether these DNAm markers are related to neural abnormalities previously identified in pediatric PTSD, which is important to identify potential 
molecular/cellular pathways associated with altered neurodevelopment contributing to pediatric PTSD (Cisler \& Herringa, 2020). In addition, previous studies examining childhood trauma are mostly based on a priori searches of candidate genes(Cecil, Zhang, \& Nolte, 2020). However, as is common with a priori searches, this approach increases the risk of confirmation biases, thereby increasing the risk of false negatives for useful epigenetic biomarkers of developmental psychopathology after adversity.

To address these knowledge gaps, we performed methylome-wide DNAm analyses using a discovery cohort of youth with PTSD, compared with two control groups. The first group (Dutch sample) consisted of trauma-exposed youth without PTSD (a resilient group), and a the second group consisted of healthy non traumatized youth. We then attempted to replicate these DNAm findings in an independent (American) cohort of youth with PTSD and a non-traumatized comparison group.

In addition to our methylome-wide analyses, we investigated whether altered methylation was further related to brain structure in regions involved in emotion regulation. This combined approach has two major advantages. First, the use of independent cohorts for discovery and replication of DNAm findings should reduce the likelihood of false positive findings and enhance generalizability of results. Second, identifying which peripherally-derived methylation differences are linked to structural brain differences in traumaexposed and PTSD youth may point to genes with the most biological relevance for pediatric trauma and PTSD through their link to central nervous system abnormalities. Given prior work implicating altered methylation in glucocorticoid pathway genes in childhood trauma and adult PTSD, we hypothesized that youth with PTSD would show altered methylation in genes annotated to the HPA axis, which would then be further associated with prefrontal and hippocampal gray matter volume abnormalities previously identified in pediatric PTSD (Jiang, Postovit, Cattaneo, Binder, \& Aitchison, 2019; Pervanidou, Makris, Chrousos, \& Agorastos, 2020). We aimed to minimize DNAm findings that were either likely to occur by chance (by requiring replication) and that may not have an impact on neural systems (by requiring association with gray matter volume). Complementing these specific hypotheses, we aimed to identify novel methylation abnormalities and methylation-brain relationships in pediatric PTSD through our epigenome-wide search.

\section{Results}

In the Dutch cohort ( $n=224), 33 \%$ of the youth were diagnosed with PTSD, 33.5\% consisted of traumatized youth without PTSD (TC) and 33.5\% consisted of healthy non-traumatized comparison (NTC) youth. Compared to the PTSD youth, TC and NTC were more likely to have a Caucasian ethnicity, and NTC were slightly younger of age (e.g. -1.3 years of difference with the PTSD youth). In the American cohort $(n=42)$, $52.4 \%$ within the USA cohort were diagnosed with PTSD, the other $47.6 \%$ consisted of NTC. In this cohort there were no significant group differences in sex, ethnicity, handedness distribution, age, or IQ. In both groups, youth with PTSD also reported comorbid internalizing and externalizing mental health problems. Demographic and mental health measures of participating youth are shown in Table 1. 
A set of epigenome-wide association study (EWAS) analyses (differentially-methylated regions [DMRs] and differentially-methylated positions [DMPs]) contrasting the PTSD and comparison groups identified statistically significant $\mathrm{CpG}$ sites. DMR analysis comparing the groups identified significant $\mathrm{CpG}$ regions at FWER < 0.05. In the Dutch cohort comparing PTSD with the NTC, the most significant effect was reported on the TNXB gene (FWER = 0.03) and the PM20D1 gene (FWER =0.04). At the TNXB gene PTSD youth showed hyper methylation compared to NTC, at the PM20D1 gene PTSD youth showed hypomethylation compared to NTC. The list of the top $10 \mathrm{CpG}$ regions in both cohorts are provided in Supplementary Table 1. The findings annotated to the TNXB and PM20D1 gene (PTSD vs. NTC youth) were replicated with the same set of epigenome wide analyses in the American cohort. Results are shown in Table 2.

In the Dutch cohort, the most significant DMP was on the corticotrophin-releasing hormone binding protein $(C R H B P)$ gene, located at cg26196496 (FDR < 0.02). Here, PTSD youth showed hyper methylation at this CpG relative to NTC youth. A plot showing greater detail on the CpGs in or near CRHBP is provided in Supplementary Figure 1. Furthermore, PTSD youth showed hypomethylation at a $\mathrm{CpG}$ site located on cg21972431 relative to trauma comparison (TC) youth (FDR $<0.01)$. These DMP findings did not replicate in the American cohort. The lists of the top $5 \mathrm{CpG}$ sites, derived from the DMP analysis in both cohorts, are shown in Supplementary Table 2.

\section{Exploratory Analysis of DMR/DMP Conjunction in the Dutch and American Cohorts}

Next, we compared the top 1000 DMRs between the two cohorts to assess potentially important DNAm abnormalities not detected by the more stringent multiple comparison correction threshold in the Dutch cohort EWAS analysis. This revealed nine DMRs that were associated with PTSD (relative to NTC youth) in both cohorts. These DMRs are shown in Table 2. In both cohorts, we identified overlap on two very large DMRs showing hypermethylation in PTSD youth relative to NTC youth (63 CpGs in the Dutch cohort, 68 CpGs in the American cohort) annotated on the TNXB gene. An overview of the DMR outcomes per cohort are shown in Supplementary Table 1. The DMP conjunction analysis did not reveal overlap between the two cohorts. The results of the DMP analyses by cohort are reported in Supplementary Table 2.

\section{Associations Between DNA Methylation and Gray Matter Volume}

We next assessed potential relationships between DNAm findings with gray matter volume in regions of interest (ROIs) previously identified in pediatric PTSD including the ventromedial prefrontal cortex, hippocampus, and amygdala (Cisler \& Herringa, 2020). We performed partial correlations to associate regional grey matter volume with peak methylation on the DMPs/DMRs that survived our multiple testing correction in the Dutch cohort or that were replicated in the American cohort. Only a subgroup of the youth participated in this post hoc analyses (see online methods).

These analyses revealed that a DMR annotated to Olfactomedin 3 (OLFM3) showed a significant negative correlation with right anterior hippocampus GMV in both cohorts (Supplementary Figure 2). Additional findings only detected in one cohort are shown in Table 3 and Supplementary Figure 2. 
All results presented above remained significant after sensitivity analyses controlling for trauma-related variables, suggesting the difference is specific to the development of pediatric psychopathology and not a consequence of trauma type, time elapsed since the trauma, and trauma load. For an overview of the outcomes see Table 3 .

\section{Discussion}

To our knowledge, this is the first study in which biological pathways of altered gene expression and brain structures implicated in pediatric PTSD have been investigated. Using two independent, international cohorts, the results of this study confirm for the first time the hypothesis that pediatric PTSD is associated with epigenetic modifications which, in turn, are associated with structural neurophenotypes implicated in pediatric PTSD. Most notably, altered DNA methylation on genes annotated to PM20D1, TNXB and OLFM3 genes were replicated across cohorts and exhibited a relationship to neural structure in at least one of the cohorts. Because this is the first genome wide methylation study in youth with PTSD to date, we cannot compare these results with other cohorts (Pervanidou et al., 2020). It is notable, however, that our results do not overlap with recent findings in adult PTSD (Smith et al. (2019). This is perhaps not surprising, given that the current study focused on a developmental sample, in which trauma load, comorbidities, substance use, and many other factors markedly differ from adult PTSD samples. However, our findings do thematically overlap with studies of childhood trauma and adult PTSD, suggesting abnormal methylation in genes annotated to the inflammation and stress response systems (Pervanidou et al., 2020).

First, changes observed on OLFM3 gene were related to hippocampal volume in both cohorts. This may represent an interaction between methylation and neuronal change related to early exposure to traumatic events and the development of PTSD at an early stage in life. OLFM3 is a neuronal protein, found throughout the brain and related to the development of microglia (Anholt, 2014; Butovsky et al., 2014). Microglia serve as key immune cells of the brain, and are activated in response to signals they receive or detect in their microenvironment. For example, they respond to injuries and infections and are able to modify the structure and function of a cell. They can for example lead to neuronal degeneration, impaired microglia activation has been linked to the development of brain disorders, such as neurodegenerative diseases (Aldana, 2019). Epigenetic mechanisms have emerged as important regulators in this process (Cheray \& Joseph, 2018). In addition to their essential role in the development of the central nervous system (CNS), microglial dysfunction is suggested to be involved in stress vulnerability and depression recurrence (Park et al., 2019). These findings are in line with our results that suggest that hyper methylation of OLFM3 may be a molecular mechanism by which hippocampal volume is decreased in youth with PTSD, potentially via enhanced CNS inflammation in affected youth.

Secondly, methylation changes in the TNXB gene were observed in both cohorts. TNXB encodes an extracellular-matrix associated glycoprotein that has been implicated in cell migration processes (ProteinAtlas), and was shown to be hypermethylated in PTSD youth relative to NTC youth. Furthermore, an additional significant effect was reported comparing PTSD with NTC youth in the Dutch cohort. This suggests that there is an effect specific to pediatric PTSD (and/or its comorbid disorders) rather than trauma-exposure generally. We also observed that in the American cohort across PTSD youth and NTC 
youth, $T N X B$ methylation is negatively associated with gray matter volume in the anterior hippocampus, originally identified in a group by age interaction in Keding \& Herringa (2015). This relationship was not observed in the Dutch cohort, however this could be due to the missing NTC in the MRI group. Interestingly, $T N X B$ has been shown to have protein-protein interactions with the protein products of VEGFA, VEGFB, NEURL 1, and NEURL $1 B$ (https://string-db.org/), all four of which have been heavily implicated in cellular processes in the anterior hippocampus. More specifically, these proteins are functionally related to hippocampal-dependent synaptic plasticity, learning, and memory (https://www.genecards.org/). This suggests that methylation of TNXB may act as a molecular mechanism of altered synaptic plasticity or growth affecting hippocampal volume in pediatric PTSD, which may then have implication for multiple hippocampal functions such as context discrimination, fear and extinction learning, and emotion regulation implicated in pediatric PTSD (Cisler \& Herringa, 2020).

In addition to the results of the DMR analysis, we detected DNAm abnormalities on the CRHBP gene in the Dutch cohort. This gene is known for its critical role in the regulation of corticotrophin-releasing hormone binding protein (CRH-BP), which in turn plays a modulatory role in the function of the HPA axis as well as $\mathrm{CRH}$ signaling within the brain. The HPA-axis is thought to play an important role in the etiology of PTSD, and the regulation of emotions and behavior (Pervanidou et al., 2020). In addition, numerous studies in rodents have shown that levels of $\mathrm{CRH}-\mathrm{BP}$ in various brain regions are highly responsive to psychological stressors (Ketchesin, Stinnett, \& Seasholtz, 2017). Furthermore, studies comparing post mortem brain tissue found that altered CRH-BP expression has been associated with adult psychopathology and suicide completion (Herringa, Roseboom, \& Kalin, 2006; Pandey, Rizavi, Bhaumik, \& Ren, 2019). In the case of pediatric PTSD, it is possible that altered CRH-BP methylation, and thus altered expression, may contribute to abnormal $\mathrm{CRH}$ signalizing both peripherally and centrally, contributing to heightened physiological and stress responses associated with PTSD. In line with earlier studies, these findings further support the involvement of abnormal gene regulation associated with HPA axis dysregulation.

While this study highlights potentially novel genetic and molecular mechanisms contributing to pediatric PTSD, several limitations should be acknowledged. First, we used data generated on different arrays between the two cohorts (HumanMethylation450 in the Dutch cohort and EPIC bead chip array in the US cohort). Data generated with these arrays are comparable (the EPIC bead chip covers the whole 450k array), but only capture a fraction of the CpG sites in the genome. Second, this study has a limited sample size in both cohorts, creating risk for both false negative and false positive findings particularly in a genetic study. To mitigate the latter, we included youth with carefully diagnosed, severe phenotypes to increase the probability that important, and true, group differences would be identified. Additionally, we applied strict corrections in the Dutch methylome-wide search, with replication in the American cohort at an uncorrected level, in order to reduce the overall risk of false positive results while balancing the risk of false negatives. Third, our study examined gene methylation using only saliva samples. Though this approach likely captures part of the underlying pathophysiology of pediatric PTSD, it is unlikely to represent exact DNA methylation in brain regions most relevant for PTSD. To mitigate this possibility, we have emphasized DNAm abnormalities that also map onto known structural brain abnormalities in pediatric PTSD. 
Future studies would be warranted to collect longitudinal data in youth with and without trauma-exposure and PTSD to determine the temporal course of methylation-neural structure relationships in relation to childhood trauma exposure and the emergence of PTSD. Given the implication of inflammatory and glucocorticoid pathways in this initial study, future studies would also benefit from including other peripheral or central markers such as cortisol and cytokine levels, and determine whether these represent mediating factors between altered peripheral methylation and structural brain changes leading to pediatric PTSD. Finally, future studies would benefit from collecting additional tissue sources (blood, saliva and eventually post-mortem brain tissue), as well as information about RNA and protein expression.

In conclusion, our findings provide new insights into the underlying biology and potential biomarkers for PTSD in youth. To our knowledge, this is the first investigation into differential DNA methylation in pediatric PTSD and its association to structural brain abnormalities. While youth with PTSD are an inherently difficult population to recruit, we utilized a combination of two independent, international, and well-phenotyped cohorts, a common data analytic pipeline, strict statistical corrections, and linkage to neural phenotypes, to increase the likelihood of important and reproducible findings in pediatric PTSD. These findings offer future targets for hypothesis driven studies in larger samples and statistical rigor for early identification of candidate gene pathways. If replicated in subsequent work, the epigenetic markers identified here could serve as novel therapeutic targets in the prevention and treatment of pediatric PTSD.

\section{Online Methods}

\section{Participants}

In the present study, two independent methylome-wide DNAm association studies were performed. The first cohort ( $N=224)$ was recruited at the Specialized Centre for Trauma and Family Treatment of the Bascule/Department of Child and Adolescent Psychiatry of the Amsterdam University Medical Center(The Netherlands). The second cohort ( $\mathrm{N}=44)$ was recruited from area mental health facilities in Madison, Wisconsin, USA. In both cohorts, medication-free youth with PTSD were included (Partial or Full PTSD diagnosis on CAPS-CA interview). They were matched for age and sex with non-traumatized, typicallydeveloping healthy comparison (NTC) youth. In the Dutch cohort an additional control group of traumatized comparison youth without PTSD (TC) was recruited. Exclusion criteria for youth with PTSD in both cohorts included imminent suicidality, history of psychotic disorder, substance abuse or dependence; IQ<70; unstable medical condition; recent use of psychotropic medication (past 4 weeks; 6 weeks for fluoxetine); MRI contraindication; and possibility of pregnancy in females. NTC and TC were screened for trauma history with use of the Children's Revised Impact of Event Scale (CRIES) and if traumatized, screened on PTSD symptoms (Perrin, Meiser-Stedman, \& Smith, 2005; Verlinden et al., 2014). NTC an TC participants were free of any history of mental illness. In both cohorts, youth with PTSD participated in ongoing studies aiming to investigate biological changes in relation to trauma exposure and PTSD. Clinical characteristics of both cohorts are shown in Table 1A and Table 1B. In the Dutch cohort, not all youth participated in the MRI analysis ( $N=52$ ). In the USA cohort, all 44 youth, including NTC youth, participated in the methylation analysis and MRI portions of the study. In both cohorts written parental consent and youth assent were 
obtained for all participants. All procedures were approved by the Medical Ethical Committee of the University Medical Center and the University of Wisconsin Health Sciences institutional review board.

\section{Clinical and Behavioral Assessments}

In both cohorts, participants and their caregivers underwent a traumatic events and psychiatric screen by trained child and adolescent psychiatrists or psychologists.

The Clinician-Administered PTSD Scale for Children and Adolescents (CAPS-CA) was used (Diehle, de Roos, Boer, \& Lindauer, 2013; Nader et al., 1996) to determine PTSD diagnosis based on DSM-IV-TR criteria. A PTSD diagnosis required at least five symptoms, including at least one from each symptom category, following Cohen and colleagues (19). In the Dutch cohort, in addition to the CAPS-CA, interview caregiver information based on the PTSD section of the Anxiety disorders interview schedule for DSM-IV (ADIS) was used (Silverman, Saavedra, \& Pina, 2001). In the USA cohort the CAPS-CA was not obtained for the first four PTSD participants included in the analysis. PTSD severity was additionally examined using the UCLA PTSD Reaction Index (PTSD-RI) (Steinberg et al., 2013). For the PTSD-RI, the greater score between the youth and caregiver report for each item was used as this was most strongly correlated with CAPS scores. In both cohorts, information about youth depressive and anxiety symptoms were measured using youth and caregiver reports. Both cohorts used different measures to assess these symptoms. In the Dutch cohort, information about internalizing and externalizing symptoms were obtained with the Revised Children's Anxiety and Depression Scale (RCADS), the Child Behavioral Checklist (CBCL) and Youth Self Report (YSR) (Achenbach, 1991; Achenbach \& Edelbrock, 1983; Chorpita, Yim, Moffitt, Umemoto, \& Francis, 2000; Kösters, Chinapaw, Zwaanswijk, van der Wal, \& Koot, 2015; Verhulst, van der Ende, \& Koot, 1997). In the American cohort, the Kiddie Schedule for Affective Disorders and Schizophrenia (KSADS) (Kaufman et al., 1997) was used for general psychiatric screening. Information about internalizing symptoms were obtained with use of the Mood and Feelings Questionnaire (MFQ) (Costello \& Angold, 1988) and Screen for Child Anxiety Related Emotional Disorders (SCARED) (Birmaher et al., 1997). To maximize comparability we provide in Table $1 \mathrm{a}$ and Table $1 \mathrm{~b}$ the rates of children that score above clinical cut-off of internalizing and externalizing symptoms based on the questionnaires described above.

\section{DNA Acquisition and Extraction}

In both cohorts three milliliters of saliva was collected and stored in Oragene DNA sample collection kits (DNA Genotek, Canada). DNA was extracted using a Gentra autopure LS system following manufacturers protocol. Genomic DNA samples were resolved on a 1\% agarose gel to verify that the DNA was of high molecular weight and quantified using Qubit (Qiagen, USA).

\section{Bisulfite Conversion and Generation of Methylation Signal}

In both cohorts, five hundred nanograms of genomic DNA was sodium bisulfite-treated for unmethylated cytosine (C) to thymine ( $\mathrm{T}$ ) conversion using the EZ DNA Methylation-Gold kit (Zymo Research). Briefly, converted DNA was amplified, fragmented, and hybridized. The converted DNA was then scanned using the HumanMethylation EPIC/850 BeadChip (Dutch cohort) and 450 BeadChip (USA cohort) following the 
manufacturer's guidelines. Illumina recently replaced the HumanMethylation450 BeadChip (450K) with the EPIC BeadChip, which nearly doubles the measured CpG sites to $>850,000$. However data obtained from two platforms is comparable within cohorts (Solomon et al., 2018).

\section{Quality Control and Data Processing}

In both cohorts, prior to hybridization, cases and controls were randomized across the 96 well plates. Technical replicates $(n=8)$ were included for quality control of array, monitoring potential batch effects. Identical analysis pipelines was implemented across cohorts using R (v.3.4.2). Each analysis step is briefly described, followed by the R package and functions used during implementation. Quality control of the raw data was performed to determine the success of the bisulfite conversion and subsequent array hybridization using Methylaid package (v.1.16.0) (Van Iterson et al., 2014). Four samples from the Dutch cohort and one sample from the American cohort were removed before further analysis during this step due to poor sample quality. Next, both the Dutch and American data sets were normalized using quantile normalization implemented in the Minfi package (v.1.22.1) (Aryee et al., 2014). Furthermore, based on Illumina's recommendations, probes present on the $X$ or $Y$ chromosomes, probes represented by a SNP, and probes known to be susceptible for cross hybridization were removed. Density plots were made to evaluate whether the normalization procedure was effective. In order to explore our datasets further, we applied a principal component analysis (PCA) on the raw and normalized datasets and we evaluated the first four components in relation to potential technical or biological confounders. Accordingly, plate batch, ethnic background (PC2 of the genetic component that is covered by the beadchip array), sex and age were selected to be included as covariates in the statistical model. Next, we identified group-related differentially methylated positions (DMPs) and regions (DMRs), using a general linear model (Imfit) that accounts for the main effects between groups: (1) PTSD vs NTC in both cohorts and (2) PTSD vs TC in the Dutch cohort. To correct for any inflation of the p-values the BACON package was used (van Iterson, van Zwet, \& Heijmans, 2017). A false discovery rate (FDR) for the DMPs and family wise error rate (FWER) for the DMRs was used to adjust for multiple testing. These corrections were applied to the results from the Dutch correct, but not the American cohort. Because the American cohort, in this case, is used as a replication sample, stringent corrections would (likely) artificially increase the rate of false negative findings beyond what would normally be considered reasonable. Therefore in the American cohort a bonferroni correction was applied combined with fisher's method for combining $p$ values from the independent tests (Chen, Liu, \& Nadarajah, 2012; Fisher, 1992). DMRs were analyzed with use of the Minfi function bumphunter. DMRs were defined to include at least 2 probes in the cluster. For each significantly associated DMR beta-values of each of the individual $\mathrm{CpGs}$ were extracted, which were used in subsequent post-hoc and sensitivity analyses. CpGs were visualized using the R package coMET (v.1.14.0) (Martin, Yet, Tsai, \& Bell, 2015).

\section{MRI Acquisition}

For the Dutch cohort all scans were acquired using a 3.0T Philips Achieva scanner (Philips Healthcare, Best, The Netherlands) equipped with a SENSE eight-channel receiver head coil. For each participant, a T1weighted structural MRI image was acquired with the following parameters: TE: $3.527 \mathrm{~ms}$, TR: $9 \mathrm{~ms}$, slice thickness: $1 \mathrm{~mm}, 170$ slices, flip angle: $8 \circ$ and image matrix $256 \times 256$ that covert the entire brain. For the 
American cohort, MRI acquisition parameters have been reported elsewhere (Keding \& Herringa 2015) and are briefly summarized here. Anatomical brain MRI was acquired using a 3.0 T GE Discovery MR750 scanner (General Electric, Milwaukee, WI) at the University of Wisconsin Department of Psychiatry. Threedimensional axial high-resolution T1 images were acquired with similar parameters as in the Dutch cohort: TE: $3.18 \mathrm{~ms}$, TR: $8.16 \mathrm{~ms}$, TI: $450 \mathrm{~ms}$, slice thickness: $1 \mathrm{~mm}, 156$ slices, flip angle: $12^{\circ}$, FOV: $25.6 \mathrm{~cm}$, and image matrix $256 \times 256$ that covered the entire brain.

\section{Image Preprocessing and Voxel-Based Morphometry}

The Dutch and American cohorts used the same image preprocessing and voxel-based morphometry (VBM) procedures, which have been reported elsewhere (Keding \& Herringa, 2015) and are briefly summarized here. Preprocessing and VBM were performed using the VBM8 toolbox (http://dbm.neuro.uni-jena.de/vbm/) in Statistical Parametric Mapping (Wellcome Department of Imaging Neuroscience, London, UK), which was executed in Matlab (Mathworks, Sherborn, MA). Standard VBM8 toolbox parameters were used for preprocessing. Images were bias corrected, tissue classified, and normalized to MNI space using linear (12parameter affine) and nonlinear transformations including high-dimensional DARTEL within a unified model. Standard values were selected to bias regularization (0.0001) and FWHM cutoff $(60 \mathrm{~mm})$. Denoising was performed with optimized Rician non-local means and HMRF weighting of 0.15 . Output-normalized GMVs were modulated for nonlinear (Jacobian) components only, resulting in images corrected for total brain volume and smoothed with a 6-mm FWHM Gaussian filter. Final voxel resolution was $1.5 \times 1.5 \times 1.5$ $\mathrm{mm}$.

\section{Post Hoc Analyses}

Peak DNA methylation at DMPs and DMRs and their associations with brain gray matter volume were assessed using partial correlation and regression analyses in $\mathrm{R}$ (ppcor) in a subset of the Dutch cohort (see Supplementary Table 3) and in the entire American cohort. Due to regulatory restrictions in the Dutch cohort it was not possible to collect neuroimaging in the non-traumatized control group. We analyzed the association with the following regions of interests (ROI's);right anterior ventromedial prefrontal cortex (vmPFC); left and right fusiform gyrus, left and right occipital cortex; right anterior hippocampus; Gray matter volume measures were extracted at the individual level from regional results reported previously in the American cohort (Keding \& Herringa, 2015).

We selected one significant DMP (significant in the Dutch cohort after multiple testing correction) and nine DMRs that showed an overlap within the two cohorts for our post-hoc analysis. Epigenome-wide replication in the exploratory EWAS analysis was defined as overlap for at least one DMP within a DMR for both cohorts, comparing the top 1000 DMRs. For the post hoc analysis we performed both partial correlation analyses between the gray matter volume data available for each cohort and the information from the original DMR. All analyses were covaried for age, sex, trauma type and PTSD duration. Additionally, these results were further corrected for multiple comparisons using an FDR correction and a Bonferroni correction for the number of post-hoc analyses conducted. Post-hoc results with a corrected $p<0.007$ (7 sets of partial correlation analyses) were considered statistically significant. 


\section{Declarations}

Acknowledgements

We would like to thank Rosanne op den Kelder (Msc), psychologist at the Bascule, Academic Centre for Child an Adolescent psychiatry, for her help with the collection of the clinical data. This project is partly funded by the Augeo Foundation. The funder has no role in the design and conduct of the study, the collection, management, analysis, or interpretation of data, or article preparation.

\section{Author Contributions}

Study concept and design: J.E, T.K., R.H., R.L, M.M.

Acquisition of data: J.E, T.K, J.Z,

Analysis and interpretation of data: J.E, T.K, P.H., R.H., J.Z, G.v.W.

Drafting of the article: J.E, T.K.,

Critical revision of the article for important intellectual content: P.H., R.H, R.L, G.v.W, C.M., M.M,

Statistical analysis: J.E, T.K., A.V., P.H.

Study supervision: R.L., R.H., M.M.

\section{References}

Achenbach, T. M. (1991). Manual for the youth self-report and 1991 profile: Department of Psychiatry, University of Vermont Burlington.

Achenbach, T. M., \& Edelbrock, C. S. (1983). Manual for the child behavior checklist and revised child behavior profile.

Aldana, B. I. (2019). Microglia-specific metabolic changes in neurodegeneration. Journal of molecular biology, 431(9), 1830-1842.

Alisic, E., Zalta, A. K., Van Wesel, F., Larsen, S. E., Hafstad, G. S., Hassanpour, K., \& Smid, G. E. (2014). Rates of post-traumatic stress disorder in trauma-exposed children and adolescents: meta-analysis. The British Journal of Psychiatry, 204(5), 335-340.

Anholt, R. (2014). Olfactomedin proteins: central players in development and disease. Frontiers in cell and developmental biology, 2, 6.

Aryee, M. J., Jaffe, A. E., Corrada-Bravo, H., Ladd-Acosta, C., Feinberg, A. P., Hansen, K. D., \& Irizarry, R. A. (2014). Minfi: a flexible and comprehensive Bioconductor package for the analysis of Infinium DNA methylation microarrays. Bioinformatics, 30(10), 1363-1369. 
Birmaher, B., Khetarpal, S., Brent, D., Cully, M., Balach, L., Kaufman, J., \& Neer, S. M. (1997). The screen for child anxiety related emotional disorders (SCARED): Scale construction and psychometric characteristics. Journal of the American Academy of Child \& Adolescent Psychiatry, 36(4), 545-553.

Butovsky, O., Jedrychowski, M. P., Moore, C. S., Cialic, R., Lanser, A. J., Gabriely, G., . . Doykan, C. E. (2014). Identification of a unique TGF- $\beta$-dependent molecular and functional signature in microglia. Nature neuroscience, 17(1), 131.

Cecil, C. A., Zhang, Y., \& Nolte, T. (2020). Childhood maltreatment and DNA methylation: A systematic review. Neuroscience \& Biobehavioral Reviews.

Chen, Z., Liu, Q., \& Nadarajah, S. (2012). A new statistical approach to detecting differentially methylated loci for case control Illumina array methylation data. Bioinformatics, 28(8), 1109-1113.

Cheray, M., \& Joseph, B. (2018). Epigenetics control microglia plasticity. Frontiers in cellular neuroscience, $12,243$.

Chorpita, B. F., Yim, L., Moffitt, C., Umemoto, L. A., \& Francis, S. E. (2000). Assessment of symptoms of DSMIV anxiety and depression in children: A revised child anxiety and depression scale. Behaviour research and therapy, 38(8), 835-855.

Cisler, J. M., \& Herringa, R. J. (2020). Posttraumatic stress disorder and the developing adolescent brain. Biological Psychiatry.

Copeland, W. E., Keeler, G., Angold, A., \& Costello, E. J. (2007). Traumatic events and posttraumatic stress in childhood. Archives of general psychiatry, 64(5), 577-584.

Costello, E. J., \& Angold, A. (1988). Scales to assess child and adolescent depression: checklists, screens, and nets. Journal of the American Academy of Child \& Adolescent Psychiatry, 27(6), 726-737.

Diehle, J., de Roos, C., Boer, F., \& Lindauer, R. J. (2013). A cross-cultural validation of the Clinician Administered PTSD Scale for Children and Adolescents in a Dutch population. European Journal of Psychotraumatology, 4(1), 19896.

Fisher, R. A. (1992). Statistical methods for research workers. In Breakthroughs in statistics (pp. 66-70): Springer.

Herringa, R. J., Roseboom, P. H., \& Kalin, N. H. (2006). Decreased amygdala CRF-binding protein mRNA in post-mortem tissue from male but not female bipolar and schizophrenic subjects.

Neuropsychopharmacology, 31(8), 1822-1831.

Jia, T., Chu, C., Liu, Y., van Dongen, J., Papastergios, E., Armstrong, N. J., . . Harris, M. (2019). Epigenomewide meta-analysis of blood DNA methylation and its association with subcortical volumes: findings from the ENIGMA Epigenetics Working Group. Molecular psychiatry, 1-12. 
Jiang, S., Postovit, L., Cattaneo, A., Binder, E. B., \& Aitchison, K. J. (2019). Epigenetic modifications in stress response genes associated with childhood trauma. Frontiers in psychiatry, 10, 808.

Kaufman, J., Birmaher, B., Brent, D., Rao, U., Flynn, C., Moreci, P., . . Ryan, N. (1997). Schedule for affective disorders and schizophrenia for school-age children-present and lifetime version (K-SADS-PL): initial reliability and validity data. Journal of the American Academy of Child \& Adolescent Psychiatry, 36(7), 980988.

Keding, T. J., \& Herringa, R. J. (2015). Abnormal structure of fear circuitry in pediatric post-traumatic stress disorder. Neuropsychopharmacology, 40(3), 537.

Ketchesin, K. D., Stinnett, G. S., \& Seasholtz, A. F. (2017). Corticotropin-releasing hormone-binding protein and stress: from invertebrates to humans. Stress, 20(5), 449-464.

Kösters, M. P., Chinapaw, M. J., Zwaanswijk, M., van der Wal, M. F., \& Koot, H. M. (2015). Structure, reliability, and validity of the revised child anxiety and depression scale (RCADS) in a multi-ethnic urban sample of Dutch children. BMC psychiatry, 15(1), 132.

Lin, D., Chen, J., Ehrlich, S., Bustillo, J. R., Perrone-Bizzozero, N., Walton, E., . . Du, Y. (2018). Cross-tissue exploration of genetic and epigenetic effects on brain gray matter in schizophrenia. Schizophrenia bulletin, 44(2), 443-452.

Liu, J., Julnes, P. S., Chen, J., Ehrlich, S., Walton, E., \& Calhoun, V. D. (2015). The association of DNA methylation and brain volume in healthy individuals and schizophrenia patients. Schizophrenia research, 169(1-3), 447-452.

Martin, T. C., Yet, I., Tsai, P.-C., \& Bell, J. T. (2015). coMET: visualisation of regional epigenome-wide association scan results and DNA co-methylation patterns. BMC bioinformatics, 16(1), 131.

McLaughlin, K. A., Koenen, K. C., Hill, E. D., Petukhova, M., Sampson, N. A., Zaslavsky, A. M., \& Kessler, R. C. (2013). Trauma exposure and posttraumatic stress disorder in a national sample of adolescents. Journal of the American Academy of Child \& Adolescent Psychiatry, 52(8), 815-830. e814.

Nader, K. O., Kriegler, J., Blake, D., Pynoos, R., Newman, E., \& Weathers, F. (1996). Clinician Administered PTSD scale for children and adolescents. National Center for PTSD.

Panagioti, M., Gooding, P. A., Triantafyllou, K., \& Tarrier, N. (2015). Suicidality and posttraumatic stress disorder (PTSD) in adolescents: a systematic review and meta-analysis. Social psychiatry and psychiatric epidemiology, 50(4), 525-537.

Pandey, G. N., Rizavi, H. S., Bhaumik, R., \& Ren, X. (2019). Increased protein and mRNA expression of corticotropin-releasing factor (CRF), decreased CRF receptors and CRF binding protein in specific postmortem brain areas of teenage suicide subjects. Psychoneuroendocrinology. 
Park, M.-J., Park, H.-S., You, M.-J., Yoo, J., Kim, S. H., \& Kwon, M.-S. (2019). Dexamethasone induces a specific form of ramified dysfunctional microglia. Molecular neurobiology, 56(2), 1421-1436.

Perrin, S., Meiser-Stedman, R., \& Smith, P. (2005). The Children's Revised Impact of Event Scale (CRIES): Validity as a screening instrument for PTSD. Behavioural and Cognitive Psychotherapy, 33(4), 487-498.

Pervanidou, P., Makris, G., Chrousos, G., \& Agorastos, A. (2020). Early Life Stress and Pediatric Posttraumatic Stress Disorder. Brain Sciences, 10(3), 169.

Ruggeri, B., Nymberg, C., Vuoksimaa, E., Lourdusamy, A., Wong, C. P., Carvalho, F. M., . . Banaschewski, T. (2015). Association of protein phosphatase PPM1G with alcohol use disorder and brain activity during behavioral control in a genome-wide methylation analysis. American Journal of Psychiatry, 172(6), 543552 .

Silverman, W. K., Saavedra, L. M., \& Pina, A. A. (2001). Test-retest reliability of anxiety symptoms and diagnoses with the Anxiety Disorders Interview Schedule for DSM-IV: child and parent versions. Journal of the American Academy of Child \& Adolescent Psychiatry, 40(8), 937-944.

Smith, A. K., Ratanatharathorn, A., Maihofer, A. X., Naviaux, R. K., Aiello, A. E., Amstadter, A. B., .. Boks, M. P. (2019). Epigenome-wide meta-analysis of PTSD across 10 military and civilian cohorts identifies novel methylation loci. BioRxiv, 585109.

Solomon, O., Maclsaac, J., Quach, H., Tindula, G., Kobor, M. S., Huen, K., ... Holland, N. (2018). Comparison of DNA methylation measured by Illumina 450K and EPIC BeadChips in blood of newborns and 14-year-old children. Epigenetics, 13(6), 655-664.

Steinberg, A. M., Brymer, M. J., Kim, S., Briggs, E. C., Ippen, C. G., Ostrowski, S. A., . . Pynoos, R. S. (2013). Psychometric properties of the UCLA PTSD reaction index: part I. Journal of traumatic stress, 26(1), 1-9.

Van Iterson, M., Tobi, E. W., Slieker, R. C., Den Hollander, W., Luijk, R., Slagboom, P. E., \& Heijmans, B. T. (2014). MethylAid: visual and interactive quality control of large Illumina 450k datasets. Bioinformatics, 30(23), 3435-3437.

van Iterson, M., van Zwet, E. W., \& Heijmans, B. T. (2017). Controlling bias and inflation in epigenome-and transcriptome-wide association studies using the empirical null distribution. Genome biology, 18(1), 19.

Verhulst, F. C., van der Ende, J., \& Koot, J. M. (1997). Youth Self-Report (YSR): Afdeling Kinder-en Jeugdpsychiatrie, Sophia Kinderziekenhuis/Academisch ....

Verlinden, E., van Meijel, E. P., Opmeer, B. C., Beer, R., de Roos, C., Bicanic, I. A., . . Lindauer, R. J. (2014). Characteristics of the Children's Revised Impact of Event Scale in a clinically referred Dutch sample. Journal of traumatic stress, 27(3), 338-344.

Walton, E., Calhoun, V., Heijmans, B., Thompson, P., \& Cecil, C. (2020). The rise of neuroimaging epigenetics: A systematic review of studies examining associations between DNA methylation and brain imaging. 


\section{Tables}

Table 1A: Demographic information, trauma history and clinical characteristics in the Dutch cohort

\begin{tabular}{|c|c|c|c|}
\hline & $\begin{array}{l}\text { PTSD youth } \\
(n=74)\end{array}$ & $\begin{array}{l}\text { Traumatized Controls } \\
(n=75)\end{array}$ & $\begin{array}{l}\text { Healthy non-traumatized } \\
\text { controls }(n=75)\end{array}$ \\
\hline $\begin{array}{l}\text { Sex } \\
\text { Boys } \\
\text { Girls }\end{array}$ & $\begin{array}{l}31(41.9 \%) \\
43 \quad(58.1 \%)\end{array}$ & $\begin{array}{l}37(49.3 \%) \\
38(50.7 \%)\end{array}$ & $\begin{array}{l}36(48.0 \%) \\
39(52.0 \%)\end{array}$ \\
\hline Age & $12.13(3.44)$ & $11.95(2.28)$ & $10.77(2.15)^{\mathrm{a}^{\star \star}}$ \\
\hline $\begin{array}{l}\text { Ethnicity } \\
\text { Caucasian } \\
\text { Other }\end{array}$ & $\begin{array}{l}44(59.5 \%) \\
30(40.5 \%)\end{array}$ & $\begin{array}{l}61(81.3 \%)^{\mathrm{c}^{\star \star}} \\
14(18.7 \%)\end{array}$ & $\begin{array}{l}64(85.3 \%)^{{ }^{\star \star}} \\
11(14.7 \%)\end{array}$ \\
\hline Index trauma & & & \\
\hline $\begin{array}{l}\text { Interpersonal } \\
\text { violence } \\
\text { Sexual abuse } \\
\text { Accidents/medical } \\
\text { trauma } \\
\text { Other }\end{array}$ & $\begin{array}{l}38(54.4 \%) \\
11(14.9 \%) \\
9(12.2 \%) \\
16(21.6 \%)\end{array}$ & $\begin{array}{l}21(35.6 \%) \\
0(0 \%) \\
37(49.3 \%) \\
17(22.7 \%)\end{array}$ & $\begin{array}{l}x \\
x \\
x \\
x\end{array}$ \\
\hline Comorbid diagnosis & & & \\
\hline $\begin{array}{l}\text { Internalizing } \\
\text { problems } \\
\text { Externalizing } \\
\text { problems }\end{array}$ & $\begin{array}{l}23(31.1 \%) \\
18(24.3 \%)\end{array}$ & $\begin{array}{l}2(2.7 \%)^{{ }^{\star \star}} \\
3(4.0 \%)^{{ }^{\star \star}}\end{array}$ & $\begin{array}{l}0(0 \%)^{\mathrm{c}^{\star \star}} \\
0(0 \%)^{\mathrm{c}^{\star \star}}\end{array}$ \\
\hline $\begin{array}{l}\text { CAPS-CA Severity } \\
\text { Score }\end{array}$ & $52.91(26.07)$ & $14.45(12.88)^{b^{\star \star}}$ & $x$ \\
\hline $\begin{array}{l}\text { CRIES-13 Severity } \\
\text { Score }\end{array}$ & $37.44(15.35)$ & $11.04(15.07)^{\mathrm{b}^{\star \star \star}}$ & $x$ \\
\hline
\end{tabular}


Continuous variables presented as mean (standard deviation); categorical variables presented as frequency (percentage). $a=$ One-way ANOVA; $b=$ Kruskal-Wallis Test; $c=$ Chi-Square Test. PTSD=post-traumatic stress disorder; CAPS-CA = Clinician Administered PTSD Scale for Children and Adolescents; CRIES-13 = Children's revised Impact of Event Scale, Revised. Comorbid internalizing and externalizing symptoms were based on the Revised Children's Anxiety and Depression Scale (RCADS; T- scores $\geq 70$ ), and the Child Behavioral Checklist (CBCL) and Youth Self Report (YSR; T score $\geq 63$ ).

Table 1B: Demographic Information, Trauma History and Clinical Characteristics in the USA cohort 


\begin{tabular}{|c|c|c|}
\hline & PTSD Youth $(n=22)$ & Healthy non-traumatized controls $(n=20)$ \\
\hline $\begin{array}{l}\text { Sex } \\
\text { Boys } \\
\text { Girls }\end{array}$ & $\begin{array}{l}8 \\
14\end{array}$ & $\begin{array}{l}7 \\
13\end{array}$ \\
\hline Age & $14.22(2.70)$ & $14.21(2.80)$ \\
\hline $\begin{array}{l}\text { Ethnicity } \\
\text { Caucasian } \\
\text { Other }\end{array}$ & $\begin{array}{l}18 \\
4\end{array}$ & $\begin{array}{l}17 \\
3\end{array}$ \\
\hline IQ & $103.2(13.0)$ & $110.5(12.5)$ \\
\hline Left Handed & 0 & 0 \\
\hline Index Trauma & & \\
\hline Interpersonal Violence & $3(13.6 \%)$ & - \\
\hline Sexual Abuse & $11(50 \%)$ & - \\
\hline $\begin{array}{l}\text { Severe Accident/medical trauma } \\
\text { Other } \\
\text { (Traumatic News, Natural Disaster) }\end{array}$ & $\begin{array}{l}3(13.6 \%) \\
5(22.7 \%)\end{array}$ & - \\
\hline Comorbid Diagnoses & & \\
\hline Depression & $19(86.4 \%)$ & - \\
\hline $\begin{array}{l}\text { Anxiety } \\
\text { ADHD }\end{array}$ & $\begin{array}{l}14(63.6 \%) \\
6(27.3 \%)\end{array}$ & - \\
\hline $\begin{array}{l}\text { Previous Psychotropic } \\
\text { Medication-Use }\end{array}$ & & \\
\hline $\begin{array}{l}\text { Depression } \\
\text { Anxiety } \\
\text { ADHD }\end{array}$ & $\begin{array}{l}8(36.4 \%) \\
3(13.6 \%) \\
7(31.8 \%)\end{array}$ & $\begin{array}{l}- \\
-\end{array}$ \\
\hline CAPS-CA Severity Score & $77.11(16.75)$ & - \\
\hline PTSD-RI Severity Score & $55.36(11.14)$ & - \\
\hline
\end{tabular}

Continuous variables presented as mean (standard deviation); categorical variables presented as frequency (percentage). There were no significant group differences (PTSD Youth vs. Healthy Youth; $p>0.05$ ) in sex, 
ethnicity, or handedness distribution, age, or IQ (based on $\mathrm{c}^{2}$ tests of independence and independent samples $t$-tests respectively). PTSD = Post-Traumatic Stress Disorder; ADHD = Attention Deficit, Hyperactivity Disorder; CAPS-CA = Clinician-Administered PTSD Scale for Children and Adolescents; PTSDRI = UCLA PTSD Reaction Index. Comorbid diagnosis were based on the Kiddie Schedule for Affective Disorders and Schizophrenia (KSADS), Mood and Feelings Questionnaire (MFQ), and Screen for Child Anxiety Related Emotional Disorders (SCARED).

\section{Table 2 was not provided with this version of the manuscript.}

Table 3: Significant post-hoc partial correlation results comparing peak methylation on DMPs/DMRs with regional grey matter volume estimates 


\begin{tabular}{|c|c|c|c|c|c|c|c|c|c|}
\hline & Gene & Chr & Region & $\begin{array}{l}r \\
\text { Dutch } \\
\text { Cohort }\end{array}$ & $\begin{array}{l}p- \\
\text { value } \\
\text { Dutch } \\
\text { Cohort }\end{array}$ & $\begin{array}{l}\text { FDR } p- \\
\text { value } \\
\text { Dutch } \\
\text { Cohort }\end{array}$ & $\begin{array}{l}\text { ÚSA } \\
\text { Cohort }\end{array}$ & $\begin{array}{l}p- \\
\text { value } \\
\text { USA } \\
\text { Cohort }\end{array}$ & $\begin{array}{l}\text { FDR p- } \\
\text { value } \\
\text { USA } \\
\text { Cohort }\end{array}$ \\
\hline \multirow{12}{*}{$\begin{array}{l}\text { PTSD } \\
\text { Youth } \\
\text { and } \\
\text { NTC } \\
\text { Youth }\end{array}$} & $C R H B P$ & 5 & $\begin{array}{l}\text { R Fusiform } \\
\text { Gyrus }\end{array}$ & 0.328 & 0.002 & 0.018 & & - & - \\
\hline & $C R H B P$ & 5 & $\begin{array}{l}\text { L Fusiform } \\
\text { Gyrus }\end{array}$ & 0.350 & $<.<001$ & 0.016 & & - & - \\
\hline & GDF7 & 2 & $\begin{array}{l}\text { R Anterior } \\
\text { Hippocampus }\end{array}$ & 0.288 & 0.006 & 0.037 & & - & - \\
\hline & GDF7 & 2 & $\mathrm{R}$ vmPFC & 0.294 & 0.005 & 0.037 & & - & - \\
\hline & GDF7 & 2 & $\begin{array}{l}\text { R Fusiform } \\
\text { Gyrus }\end{array}$ & - & - & - & 0.455 & $<.001$ & 0.003 \\
\hline & GDF7 & 2 & $\begin{array}{l}\text { L Fusiform } \\
\text { Gyrus }\end{array}$ & - & - & - & 0.375 & 0.003 & 0.018 \\
\hline & OLFM3 & 1 & $\begin{array}{l}\text { R Anterior } \\
\text { Hippocampus }\end{array}$ & -0.323 & 0.002 & 0.018 & -0.453 & <. 001 & 0.003 \\
\hline & OLFM3 & 1 & R vmPFC & -0.369 & $<.001$ & 0.014 & - & - & - \\
\hline & PM20D1 & 1 & $\begin{array}{l}\text { R Fusiform } \\
\text { Gyrus }\end{array}$ & - & - & - & 0.384 & 0.003 & 0.017 \\
\hline & SLC1A4 & 2 & $\begin{array}{l}\text { R Fusiform } \\
\text { Gyrus }\end{array}$ & - & - & - & 0.511 & $<.001$ & 0.001 \\
\hline & SLC1A4 & 2 & $\begin{array}{l}\text { L Fusiform } \\
\text { Gyrus }\end{array}$ & - & - & - & 0.335 & 0.010 & 0.043 \\
\hline & $T N X B$ & 6 & $\begin{array}{l}\text { R Anterior } \\
\text { Hippocampus }\end{array}$ & - & - & - & -0.406 & 0.001 & 0.012 \\
\hline
\end{tabular}

Partial correlations were controlled for age and sex of participant. All findings met threshold of FDR $p$-value $<0.05$. BOLD indicates a significant result in both cohorts; $\mathrm{Chr}=$ Chromosome; $\mathrm{DMR}=$ Differently methylated regions; DMP = Differently methylated position; PTSD = Post-Traumatic Stress Disorder; NTC = Non-Traumatized Control; vmPFC = ventromedial prefrontal cortex

\section{Supplementary Files}

This is a list of supplementary files associated with this preprint. Click to download.

- 20201002supplTable1PediatricPosttraumaticStressEnsink.docx

- 20201002supplTable1PediatricPosttraumaticStressEnsink.docx

- 20201002supplTable2PediatricPosttraumaticStressEnsink.docx 
- 20201002supplTable2PediatricPosttraumaticStressEnsink.docx

- 20201002supplTable3PediatricPosttraumaticStressEnsink.docx

- 20201002supplTable3PediatricPosttraumaticStressEnsink.docx

- 20201002supplFigure1PediatricPosttraumaticStressEnsink.docx

- 20201002suppIFigure1PediatricPosttraumaticStressEnsink.docx

- 20201002supplFigure2PediatricPosttraumaticStressEnsink.docx

- 20201002supplFigure2PediatricPosttraumaticStressEnsink.docx 